degree of harm, whether physical, psychological or social. The balancing of harm against benefit is the risk-benefit ratio, and a favourable risk-benefit ratio is a prime determining factor of whether a research study should be allowed to go ahead. However, this framework is challenged when it comes to HPSR studies, because, while the risks may accrue to one group, the benefit may accrue to another. Clinical equipoise is "traditionally defined as a state of genuine uncertainty on the relative value of two approaches being compared in a trial" (3). In HPSR studies, clinical equipoise is difficult to assess since the intervention is not about evaluating its efficacy, but to "analyse changes in policy, implementation or service delivery" (1: p 6). As "risk" is not clearly spelt out in HPSR studies, it gets interpreted along the lines of risk in biomedical research; but as the author of the above case study suggests, the concept of risk needs to be rearticulated for HPSR studies.

\section{References}

1. Alliance for Health Policy and Systems Research (WHO) and Global Health Ethics Unit (WHO). Ethical considerations for health policy and systems research. Geneva, WHO; 2019[cited 2020 Jan 26] Available from: https:// apps.who.int/iris/bitstream/handle/10665/330033/9789241516921eng.pdf?ua $=1$

2. Mohan M. Autonomy and risk in health policy and systems research. Indian J Med Ethics. 2020 Jan-Mar;5(1) NS:13-4. DOI: 10.20529/ IJME.2020.010

3. Rabinstein AA, Brinjikji W, Kallmes D F. Equipoise in clinical trials: Angst and progress. Circ Res. 2016 Sep 16; 119(7):798-800. DOI: 10.1161/ CIRCRESAHA.116.309594.

\title{
Observation of unsafe medical practice during research in a healthcare- deprived area
}

\section{BEVIN VIJAYAN}

Keywords: Unethical treatment, quacks, RMPs, reporting unethical treatment

\section{Background and context}

The health workforce in India is unevenly distributed within and across regions, with qualified practitioners more concentrated in urban areas (1). In rural and poor areas where there are no doctors, unqualified medical practitioners are the first point of contact for healthcare needs (1). These unqualified medical practitioners, also called Rural Medical Practitioners (RMP), practise western medicine without any formal training. They may hold certificates from organisations that are not recognised by regulatory authorities (2). RMPs work under doctors as helpers, observe their work, and then set up clinics or practise in areas where there are no doctors (3). They are described as quacks by the IMA and the Supreme Court of India. The IMA has been fighting to eliminate these providers from the system $(3,4)$.

An ethnographic ${ }^{1}$ study was done among a nomadic tribe in a rural district to understand their access to healthcare. The tribe lives in settlements called tandas (5). The nearest public health facility from this particular tanda is around $9 \mathrm{~km}$. Road connectivity to this public health facility is good, but there is

\footnotetext{
Author: Bevin Vijayan (bevinvinay@gmail.com), PhD Scholar, Achutha Menon Centre for Health Science Studies, Sree Chitra Tirunal Institute for Medical Sciences and Technology, Thiruvananthapuram, Kerala, 695011 INDIA.

To cite: Vijayan B. Observation of unsafe medical practice during research in a healthcare-deprived area. Indian J Med Ethics. 2020 Jan-Mar; 5(1) NS: 16-7. DOI:10.20529/IJME.2020.012.

CIndian Journal of Medical Ethics 2020
}

no public transport to reach the facility from the tanda. People have to rely on private vehicles. A few RMPs and Bachelor of Ayurveda Medicine and Surgery doctors live in a settlement about $3 \mathrm{~km}$ from the tanda. They provide services in their clinic and also visit the tanda whenever called. They are available on call even in the middle of the night.

\section{The case}

The researcher, who is undertaking an ethnographic study, visits the tanda regularly to observe and interview the people regarding their access to healthcare. Consent has been obtained from the village head and the elected representative to carry out this work. During one such visit, the researcher observes an old man being treated by an RMP for a head injury sustained by a fall. The RMP does not belong to the tanda but provides emergency services here and was called by the relatives. The RMP informs the family that the wound needs suturing to control bleeding. The researcher notes that the RMP instructs one of the relatives to bring a bowl of hot water and some old newspapers. The RMP then takes out a pair of gloves, cotton and suturing materials from plastic boxes in a compartment of his bag. The boxes do not seem to have been maintained in aseptic conditions. The RMP uses the cotton to clean the wound, asking the relatives to hold the old man's body and head in position. He sutures the wound without administering local anaesthetic or other pain medication. The researcher observes the old man writhing in pain throughout the procedure. After suturing, the RMP cuts the suture material off with a pair of scissors which he takes out from his bag.

After the procedure, the RMP instructs the relatives to clean the scissors, using the hot water provided, and asks them to dispose of the used cotton and gauze which was placed on 
the old newspaper. He then washes his hands and puts the scissors and suturing materials back in the plastic box.

The RMP takes a syringe from the bag, loads it with what seems to be some medicine, and injects it into the old man's buttocks. He then removes the needle and flushes the syringe by loading it with the water from a container nearby. He then takes another vial from his bag, attaches the needle to it, loads the syringe with the medicine from the vial, and injects it into the other buttock. The syringe is put back into the bag. The RMP informs the family that he will visit them in the evening to inspect the wound. He gives them antibiotics and pain killers and instructs them on their use. He also instructs the family to get a barber and shave around the wound area.

The researcher, who is trained in modern medicine, finds the treatment inappropriate. If he intervenes in this situation, the family's only alternative would be to take the old man to the hospital $9 \mathrm{~km}$ away. Reporting the RMP to the concerned authorities would deprive the tanda of the only source of healthcare available at their doorstep.

\section{Ethical issues}

1. What are the researcher's ethical responsibilities? What are his possible courses of action, and why?

2. The researcher obtained consent from the village representatives to observe the residents of the tanda. Should the researcher have obtained consent from the RMP, who is not from the tanda, to observe his treatment of the old man?

3. The researcher on his next visit in the tanda learns that the old man had died on the fifth day after his fall. Considering the events that the researcher observed, how would the death of the old man reflect on the dilemmas already faced by the researcher?

Acknowledgements: The author thanks Dr Vijayaprasad Gopichandran, Dr Amar Jesani, Dr Rakhi Ghoshal and Dr Ravi Prasad Verma for their comments during the Workshop on Public Health Case Studies held on March 26-27, 2018 at the Achutha Menon Centre for Health Science Studies, SCTIMST, Thiruvananthapuram.

\section{Note}

1 Ethnography is a research tool which describes and interprets the shared and learned patterns of behaviour, beliefs and languages of a group. The aim of ethnography is to provide rich insights and descriptions into the views, beliefs and actions of people through detailed observations and interviews. It involves observations of the group and interviews of participants.

\section{References}

1. Rao $M$, Rao KD, Kumar AS, Chatterjee $M$, Sundararaman T. Human resources for health in India. Lancet. 2011 Feb 12;377(9765):587-98.Doi: 10.1016/S0140-6736(10)61888-0. Epub 2011 Jan 10.

2. George A, lyer A. Unfree markets: Socially embedded informal health providers in northern Karnataka, India. Soc Sci Med. 2013 Nov; 96:297304. doi: 10.1016/j.socscimed.2013.01.022.

3. Pulla P. Are India's quacks the answer to its shortage of doctors? BMJ. 2016 Jan 21;i291. Doi: https://doi.org/10.1136/bmj.i291

4. Nandan D, Agarwal D. Human resources for health in India: Urgent need for reforms. Indian J Community Med. 2012 Nov 15;37(4):205. DOI: 10.4103/0970-0218.103464

5. Roy Burman JJ. Chapter 3, Laman Banjaras: The people. In: Ethnography of a denotified tribe: The Laman Banjaras. New Delhi: Mittal Publications; 2010; pp19-24.

\section{The non-formal healthcare provider dilemma - to hate the player or the game?}

\section{RAVI PRASAD VARMA}

Keywords: Informal care provider, harm, professional codes, research objectivity, moral dilemma

The case study by Bevin Vijayan (1) draws attention to a wellrecognised public health problem - trauma and the lack of formal trauma care services in India (2). This is the reality in

Author; Ravi Prasad Varma (rpvarma@sctimst.ac.in), Associate Professor, Sree Chitra Tirunal Institute for Medical Sciences and Technology, Achutha Menon Centre for Health Science Studies, Trivandrum, INDIA.

To cite: Varma RP. The non-formal health care provider dilemma - to hate the player or the game? Indian J Med Ethics. 2020 Jan-Mar;5(1) NS: 17-9. DOI: 10.20529/IJME.2020.013.

Peer Reviewer: An anonymous reviewer

OIndian Journal of Medical Ethics 2020
India for trauma and for many other health problems. Despite decades of dutifully repeating the platitudes of "availability" and "accessibility", the problem is not merely a case of a service being "present" or "absent". A layered milieu of multiple elements related to peoples, systems and cultures determines the services people actually receive. It is here that we see the non-formal healthcare provider, often called a Rural Medical Practitioner (RMP), make an entrance.

Do the practices of these RMPs amount to biomedicine? Biomedically speaking, one would approach the incident referred to in the case study with several concerns. Why did the person fall in the first place? Is it due to an underlying endocrine or metabolic disorder, or perhaps a stroke? What are the possible issues the person has in addition to the scalp laceration - other 